\title{
Nitro-fatty acids decrease type I interferons and monocyte chemoattractant protein 1 in ex vivo models of inflammatory arthritis
}

\author{
A. L. Hansen ${ }^{1 \dagger}$, L. S. J. Rahbek ${ }^{1 \dagger}$, A. S. Sørensen ${ }^{1}$, M. P. Hundahl' ${ }^{1}$, S. Lomholt ${ }^{1}$, C. K. Holm ${ }^{1}$ and \\ Tue W. Kragstrup ${ }^{1,2,3^{*}}$
}

\begin{abstract}
Background: Inflammatory arthritis including rheumatoid arthritis (RA) and spondyloarthritis (SpA) is characterized by inflammation and destruction of the joints. Approximately one third of patients do not respond to first-line treatments. Nitro-fatty acids are bioactive lipids with anti-inflammatory properties and tissue-protective functions. The nitro-fatty acid 10- $\mathrm{NO}_{2}$-oleic acid (10- $\left.\mathrm{NO}_{2}-\mathrm{OA}\right)$ is being tested in clinical trials for patients with fibrotic and inflammatory conditions. Here, we tested whether $10-\mathrm{NO}_{2}-\mathrm{OA}$ could inhibit immune reactions involved in the inflammatory and joint destructive processes in inflammatory arthritis.
\end{abstract}

Methods: Synovial fluid and blood samples were obtained from 14 patients with active RA or SpA. The in vitro models consisted of synovial fluid mononuclear cells (SFMCs) cultured for 48 h, SFMCs cultured for 21 days, and fibroblastlike synovial cells (FLSs) co-cultured with peripheral blood mononuclear cells (PBMCs) for $48 \mathrm{~h}$. Cells were treated with or without 10- $\mathrm{NO}_{2}-\mathrm{OA}$ or the tumor necrosis factor alpha (TNFa) inhibitor etanercept. Supernatants were analyzed for type I interferon, monocyte chemoattractant protein-1 (MCP-1), matrix metalloproteinase 3 (MMP3) and tartrate resistant acid phosphatase (TRAP).

Results: In SFMCs cultured for $48 \mathrm{~h}, 10-\mathrm{NO}_{2}-\mathrm{OA}$ dose-dependently decreased the secretion of bioactive type I interferons and MCP-1 but not MMP3 ( $P=0.032, P=0.0001$, and $P=0.58$, respectively). Both MCP-1 and MMP3 were decreased by etanercept $\left(P=0.0031\right.$ and $P=0.026$, respectively). In SFMCs cultured for 21 days, 10- $\mathrm{NO}_{2}-\mathrm{OA}$ significantly decreased the production of MCP-1 but not TRAP $(P=0.027$ and $P=0.1523$, respectively). Etanercept decreased the production of TRAP but not MCP-1 $\left(P<0.001\right.$ and $P=0.84$, respectively). In co-cultures of FLSs and PBMCs, $10-\mathrm{NO}_{2}-$ OA decreased the production of MCP-1 $(P<0.0001)$. This decrease in MCP-1 production was not seen with etanercept treatment $(P=0.47)$.

Conclusion: $10-\mathrm{NO}_{2}-\mathrm{OA}$ decreased the release of MCP-1 in three models of inflammatory arthritis. Of particular interest, 10- $\mathrm{NO}_{2}-\mathrm{OA}$ inhibited type I interferon, and 10- $\mathrm{NO}_{2}-\mathrm{OA}$ was more effective in reducing MCP-1 production in cultures dominated by FLSs compared with etanercept. Our results encourage clinical investigations of $10-\mathrm{NO}_{2}-\mathrm{OA}$ in patients with inflammatory arthritis.

Keywords: Nitro-fatty acid, Immunosuppressive drug, Antirheumatic drug, Inflammation, Autoimmunity, Arthritis

*Correspondence: kragstrup@biomed.au.dk

${ }^{\dagger}$ A. L. Hansen and L. S. J. Rahbek have contributed equally to this work

${ }^{1}$ Department of Biomedicine, Aarhus University, Høegh-Guldbergs Gade

10, C. F. Møllers Allé 6, 8000 Aarhus C, Denmark

Full list of author information is available at the end of the article

\section{Introduction}

Rheumatoid arthritis (RA), psoriatic arthritis, and spondyloarthritis (SpA) belong to the group of inflammatory arthritis, which is a group of diseases characterized 
by synovitis and cartilage and bone destruction. Early immune suppression with disease modifying antirheumatic drugs (DMARDs) has drastically improved the management of inflammatory arthritis [1]. However, approximately one third of patients do not respond to first-line treatments, and some patients have inadequate responses to several different drugs [2]. Further, current treatments only dampen dsease activity and do not lead to cure of disease. For this reason, there is an ongoing search for new ways to suppress pathogenic mechanisms in these diseases.

Approximately 20 years ago, a new group of bioactive lipids with anti-inflammatory properties and tissue-protective functions was discovered [3]. These nitro-fatty acids are formed upon a non-enzymatic nitration by nitrogen dioxide $\left(\mathrm{NO}_{2}\right)$ of unsaturated fatty acids [4] (such as conjugated linoleic acid [5] and oleic acids). The nitro-fatty acids are formed endogenously under basal metabolic conditions in humans and levels are increased during various inflammatory conditions [6-8] and infection [9]. Nitro-fatty acids can posttranslationally modify target proteins through Michael addition reactions resulting in $S$-nitro-alkylations at available cysteines [10]. This leads to anti-inflammatory and antioxidative changes via modulation of downstream signaling events in pathways such as nuclear factor- $\mathrm{kB}$ (NFKB) [10], peroxisome proliferator-activated receptor $\gamma$ (PPAR $\gamma)[11,12]$, and nuclear factor erythroid 2-related factor 2/Kelch- like erythroid cell-derived protein with $\mathrm{CNC}$ homology-associated protein 1 (Nrf2/Keap1) [13]. 10-nitro oleic acid (10- $\mathrm{NO}_{2}$ $\mathrm{OA}$ ) is now being studied in fibrotic and inflammatory conditions. The drug has been shown to be safe and welltolerated in a phase 1 study [14] and is being investigated in glomerulosclerosis (NCT03422510) and kidney injury (NCT02248051).

Recently, nitro-fatty acids have been shown to inhibit the production of type I interferons via stimulator of interferon genes (STING) [9]. STING is essential for the production of type I interferon in the host response to infections with DNA viruses [15-17] and bacteria [18-21]. STING requires posttranslational palmitoylation at two conserved cysteine residues (cysteine 88 and cysteine 91) for its activation, signaling and subsequent type I interferon response [22]. Nitro-fatty acids have been shown to nitroalkylate the thiol group of exactly these two cysteine residues in STING. This inhibits the downstream STING-signaling which in turn inhibits the production of interferons $[9,23]$.

Autocrine type I interferon signalling in dendritic cells can cause an inflammatory arthritis phenotype in mice [24] and the interferon signature is increased in early arthritis [25]. Therefore, targeting the production of interferons might serve as a potential treatment of inflammatory arthritis. A variety of other cytokines, chemokines and enzymes are involved in the arthritogenic processes of inflammatory arthritis [26]. The chemokine monocyte chemoattractant protein-1 (MCP-1) is increased and acts as a significant mediator of inflammation and macrophage infiltration in the joint $[27,28]$. Matrix metalloproteinase 3 (MMP3) can cleave the proteoglycan part of articular cartilage [29] and tartrate resistant acid phosphatase (TRAP) is produced by osteoclasts resulting in bone resorption [30].

Here, we investigate the effect of $10-\mathrm{NO}_{2}-\mathrm{OA}$ on the production of type I interferons, MCP-1, MMP3, and TRAP in ex vivo models mimicking the different components of the disease processes in inflammatory arthritis. 10- $\mathrm{NO}_{2}$-OA showed an inhibitory effect on type I interferon production. In all three models of inflammatory arthritis, $10-\mathrm{NO}_{2}-\mathrm{OA}$ treatment resulted in a decrease in MCP-1 production. There was no effect of $10-\mathrm{NO}_{2}-\mathrm{OA}$ on MMP3 or TRAP secretion.

\section{Materials and methods Patients and samples}

A cross-sectional, paired set of synovial fluid mononuclear cells (SFMCs) and peripheral blood mononuclear cells (PBMCs) were obtained from patients with chronic RA $(n=8)$ or peripheral SpA $(n=6)$ with at least one swollen joint (from where synovial fluid was acquired) at the outpatient clinic at Aarhus University Hospital at the time of therapeutic arthrocentesis as previously described (Table 1) [31-33]. Not all patient samples were used in all experiments. The exact number of patients in each experiment has been stated in

Table 1 Patient characteristics

\begin{tabular}{ll}
\hline Age (years) & $37(29-60)$ \\
Gender (female) & 8 \\
Diagnosis & 8 \\
$\quad$ Rheumatoid arthritis & 6 \\
$\quad$ Peripheral spondyloarthritis & $6.5(1.5-19)$ \\
Disease duration (years) & \\
Treatment & 6 \\
Methotrexate & 3 \\
Sulfasalazine & 3 \\
TNF inhibitor & \\
Disease activity & 20.0 (6.5-55.0) \\
CRP (mg/L) & $3.56(3.1-4.5)$ \\
DAS28CRP (0-10) & \\
\hline
\end{tabular}

Data are expressed as median (interquartile range). Information on disease duration, treatment, and disease activity was missing in 4 patients. TNF; tumor necrosis factor; C-reactive protein; DAS28CRP, Disease activity score based on CRP and number of tender and swollen joints in 28 joints 
the figure legends and shown in Additional file 1: Fig. S1.

\section{Culture conditions and read out}

The nitrated oleic acid $10-\mathrm{NO}_{2}-\mathrm{OA}$ (Cayman Chemicals) was used at $5 \mu \mathrm{M}$ and $10 \mu \mathrm{M}$ diluted in ethanol (same concentratiosn as used previously) [9]. In all experiments, cells cultured with the TNF $\alpha$ inhibitor etanercept at $5 \mu \mathrm{g} /$ $\mathrm{ml}$ was used as a treatment control (same concentratiosn as used previously) [33]. Untreated cells or cells treated with ethanol (vehicle) were used as matched negative controls. Supernatants were harvested after centrifugation of the culture plates at $1200 \mathrm{rpm}$ for $5 \mathrm{~min}$ and the cell-free supernatant was thereafter stored at $-80{ }^{\circ} \mathrm{C}$ for later analysis. Results with the etanercept treatment control has partially been published previously together with another dataset [33]. An MTT assay was used to measure cell viability (Roche) (Additional file 1: Fig. S2).

\section{SFMC 48-h ex vivo model}

The 48-h culture was used as an in vitro model of inflammatory arthritis dominated by lymphocytes and monocytes. SFMCs were isolated and cultured as described previously $(n=12)$ [31]. Briefly, SFMCs were isolated by Ficoll-Paque (GE Healthcare) density-gradient centrifugation and frozen at $-135{ }^{\circ} \mathrm{C}$. The cells were then thawed and seeded at a concentration of $1 \times 10^{6}$ cells/ $\mathrm{ml}$ in Dulbecco's modified Eagle's medium with $10 \%$ fetal calf serum, penicillin, streptomycin, and glutamine (culture medium) for $48 \mathrm{~h}$ and kept in a humidified incubator at $37{ }^{\circ} \mathrm{C}$ and $5 \% \mathrm{CO}_{2}$. After $48 \mathrm{~h}$, the cell-free culture supernatants were analyzed for the concentration of type I interferons, MCP-1, and MMP3 (Additional file 1: Fig. S3).

\section{SFMC 21-day ex vivo model}

The 21-day culture was used as a model of low-grade inflammatory osteoclastogenesis [34]. The cultures were performed as described for the 48-h model, changing the medium and renewal of incubation condition every 2-3 days for a total of 21 days $(n=9)$ [31]. This means that cultures were either untreated, or treated with ethanol control, $10-\mathrm{NO}_{2}$-OA $5 \mu \mathrm{M}$ or $10 \mu \mathrm{M}$, or etanercept for the entire period. Culture supernatants were analyzed for the concentration of MCP-1 and the enzyme activity of tartrate-resistant acid phosphatase (TRAP) (Additional file 1: Fig. S3).

\section{FLS-PBMC 48-h ex vivo model}

FLSs were grown from SFMCs and used for analyses at passage $4-5$ as previously described [35]. The FLS-PBMC co-culture was used as an in vitro model of inflammatory arthritis modelling the interactions between FLSs and mononuclear cells migrating to the inflamed joint. Briefly, FLSs were grown from SFMCs changing the culture medium every 2-3 days. When the cell layer was $70 \%$ confluent after approximately 14 days, the FLSs were passaged by trypsin/EDTA treatment and used for analyses at passage $4-5$. To start the co-culture, the FLSs were cultured with paired autologous PBMCs in culture medium for $48 \mathrm{~h}(\mathrm{n}=6)$. Culture supernatants were analyzed for the concentration of MCP-1 and MMP3 (Additional file 1 Fig. S4).

\section{MCP-1 and MMP3 ELISA and TRAP measurement}

The concentration of MCP-1 (Biolegend, San Diego, CA, USA) and MMP3 (R\&D Systems) was analyzed by commercially available enzyme-linked immunosorbent assays (ELISA) according to the manufacturer's instructions. TRAP was analyzed by an enzymatic assay (B-bridge International, CA, USA) according to the manufacturer's instructions as done previously [34].

\section{HEK-Blue interferon- $\alpha / \beta$ Cell assay}

Human type I interferon bioactivity was analyzed by utilizing the commercially available reporter cell line HEK-Blue interferon- $\alpha / \beta$ (InvivoGen) according to the manufacturer's instructions. SEAP levels were determined using a spectrophometer at OD $620 \mathrm{~nm}$.

\section{Statistics}

Statistical analyses and graphs were done using GraphPad Prism 9 for Mac (GraphPad Software, La Jolla, CA, USA). All data were transformed to ratios by dividing the value of the samples with the value of either untreated cultures (etanercept) or ethanol treated cultures (10-NO2-OA). Ratios were then log transformed and analyzed with repeated measures one-way ANOVA (testing for dose-response relationship) or the paired t-test (comparing treatment group with negative control group). A two-sided $P$ value $<0.05$ was considered statistically significant.

\section{Results}

10- $\mathrm{NO}_{2}-\mathrm{OA}$ decreases bioactive type I interferon production by SFMCs cultured for $48 \mathrm{~h}$

Nitro-fatty acids have previously been shown to function via inhibition of type I interferon production. Therefore, the secretion of type I interferons by SFMCs cultured for $48 \mathrm{~h}$ was assessed. The $10-\mathrm{NO}_{2}-\mathrm{OA}$ significantly decreased bioactive type I interferons dose-dependently $(P=0.032)$. The decrease was not significant when 


\section{Type I interferon SFMC 48 hour culture}

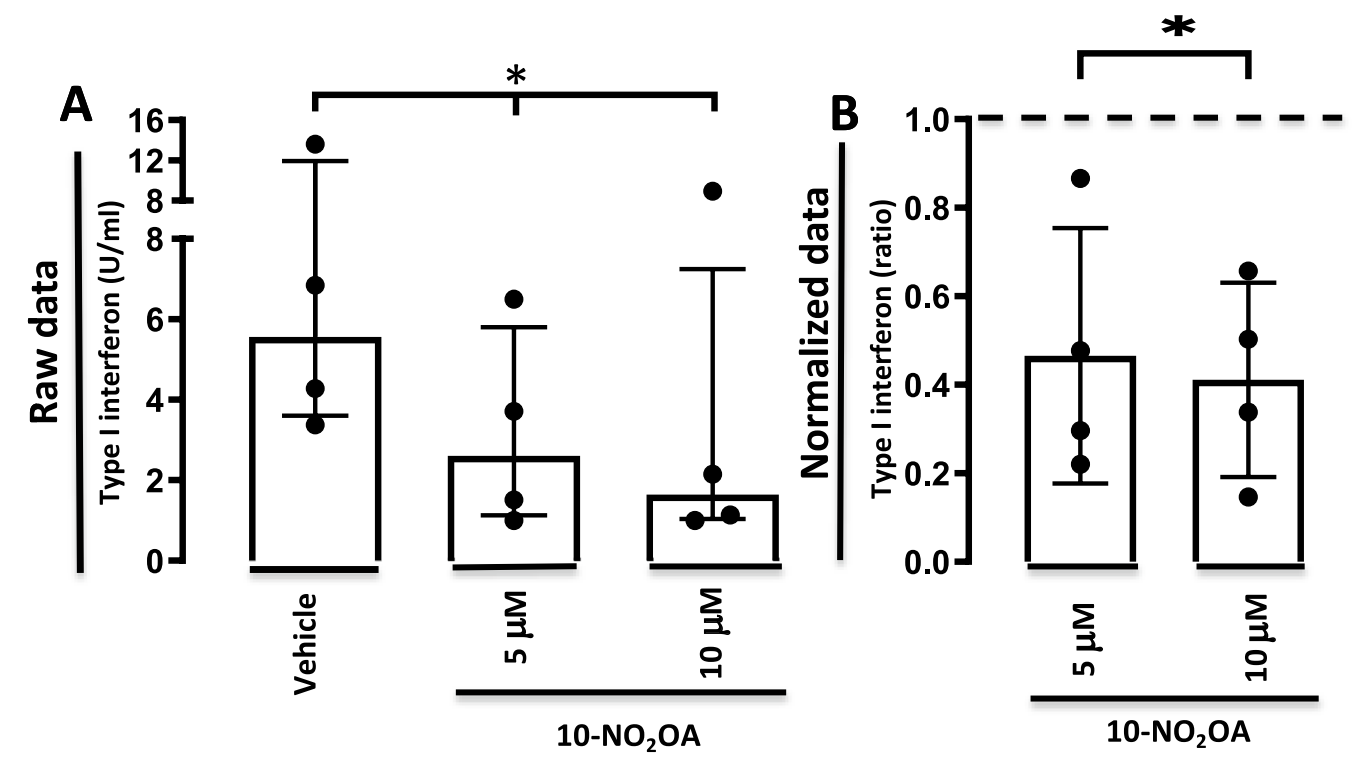

Fig. 1 Type I interferon bioactivity in SFMC 48-h cultures incubated with vehicle or 10- $\mathrm{NO}_{2}-\mathrm{OA}$ at $5 \mu \mathrm{M}$ or $10 \mu \mathrm{M}$. A Data shown as type I interferon bioactivity $(\mathrm{U} / \mathrm{ml})$. Bars indicate median and interquartile range. $\mathbf{B}$ Data shown as ratios of the type I interferon bioactivity in treated cultures divided by the bioactivity in the control culture. Dotted line represent the type I interferon bioactivity in the control culture. Bars indicate mean and SD. ${ }^{*}=P<0.05$

separately comparing cultures treated with $10-\mathrm{NO}_{2}$-OA either at $5 \mu \mathrm{M}(P=0.057)$ or at $10 \mu \mathrm{M}(P=0.052)$ and the negative control cultures treated with ethanol (Fig. 1).

\section{$10-\mathrm{NO}_{2}-\mathrm{OA}$ reduces MCP-1 but not MMP3 production by SFMCs cultured for $\mathbf{4 8} \mathrm{h}$}

We then tested whether $10-\mathrm{NO}_{2}-\mathrm{OA}$ affects the secretion of the proinflammatory chemokine MCP-1 by SFMCs. The $10-\mathrm{NO}_{2}-\mathrm{OA}$ significantly decreased the production of $\mathrm{MCP}-1$ both at $5 \mu \mathrm{M}(P=0.0015)$, at $10 \mu \mathrm{M}(P=0.0006)$ and in a dose dependent manner $(P=0.0001)$. Etanercept also significantly decreased the production of MCP-1 $(P=0.0031)$ (Fig. 2). We then tested whether $10-\mathrm{NO}_{2}-\mathrm{OA}$ affects the secretion of the connective tissue matrix degrading enzyme MMP3 by SFMCs. The $10-\mathrm{NO}_{2}-\mathrm{OA}$ did not alter the production of MMP3 at neither the $5 \mu \mathrm{M}$ concentration $(P=0.36)$, the $10 \mu \mathrm{M}$ concentration $(P=0.39)$ nor dose-dependently $(P=0.58)$. In contrast, etanercept significantly decreased the production of MMP3 $(P=0.026)$ (Fig. 2).

\section{0- $\mathrm{NO}_{2}-\mathrm{OA}$ reduces $\mathrm{MCP}-1$ production}

\section{but not inflammatory osteoclastogenesis in SFMCs} cultured for $\mathbf{2 1}$ days

Then, the effect of $10-\mathrm{NO}_{2}-\mathrm{OA}$ on the secretion of TRAP and MCP- 1 by SFMCs cultured for 21 days was studied to evaluate the immune modulatory capacity of $10-\mathrm{NO}_{2}-$ $\mathrm{OA}$ in this model of inflammatory osteoclastogenesis.
The $10-\mathrm{NO}_{2}-\mathrm{OA}$ did not alter the production of TRAP at neither the $5 \mu \mathrm{M}$ concentration $(P=0.31)$, the $10 \mu \mathrm{M}$ concentration $(P=0.11)$ nor dose-dependently $(P=0.15)$. Etanercept significantly reduced the production of TRAP $(P=0.0001)$ (Fig. 3$)$. The $10-\mathrm{NO}_{2}$-OA significantly decreased the production of MCP-1 at $10 \mu \mathrm{M}(P=0.029)$ and in a dose dependent manner $(P=0.027)$. There was no statistically significant reduction in cultures treated with $10-\mathrm{NO}_{2}-\mathrm{OA}$ at $5 \mu \mathrm{M}(P=0.17)$. There was no difference between cultures treated with etanercept and the negative untreated control cultures $(P=0.84)$ (Fig. 3).

\section{$10-\mathrm{NO}_{2}-\mathrm{OA}$ reduces $\mathrm{MCP}-1$ production but not MMP3} production by co-cultures of FLSs and autologous PBMCs Next, the effect of $10-\mathrm{NO}_{2}-\mathrm{OA}$ was evaluated in the cocultures of FLSs and autologous PBMCs. The $10-\mathrm{NO}_{2}-\mathrm{OA}$ significantly decreased the production of MCP-1 both at $5 \mu \mathrm{M}(P=0.014)$, at $10 \mu \mathrm{M}(P=0.0089)$ and in a dose dependent manner $(P<0.0001)$. This decrease in MCP-1 production was not seen with etanercept treatment $(P=0.47)$. Neither $10-\mathrm{NO}_{2}$-OA $(P=0.31)$ nor etanercept $(P=0.24)$ decreased the production of MMP3 by the cocultures of FLSs and autologous PBMCs (Fig. 4).

\section{Discussion}

Approximately one third of patients with inflammatory arthritis do not benefit from treatment with current available first-line DMARDs. Therefore, there is a 


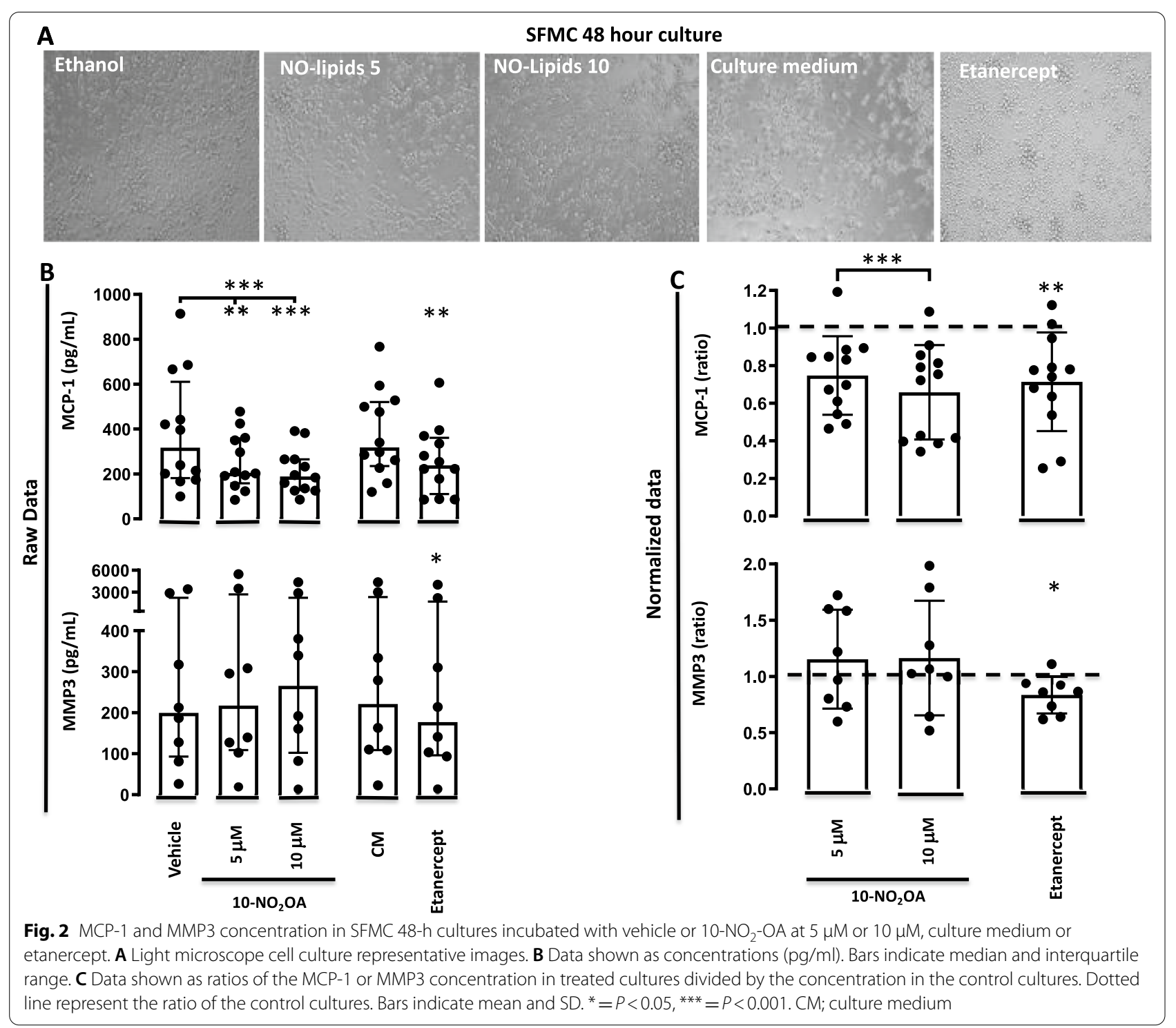

need for target validation and further drug development to improve therapeutic management of these patients. Nitro-fatty acids modify inflammatory pathways and $10-\mathrm{NO}_{2}$-OA is now being studied as a therapeutic drug in glomerulosclerosis and kidney injury. In this study, we tested whether $10-\mathrm{NO}_{2}$-OA decreases the production of type I interferons, MCP-1, MMP3, and TRAP in ex vivo models mimicking the different components of the disease process in inflammatory arthritis.

Recently, nitro-fatty acids were shown to decrease type I interferon production by directly targeting STING activation and signaling [9]. Therefore, we first tested whether $10-\mathrm{NO}_{2}$-OA could alter the production of type I interferons in SFMCs from inflammatory arthritis patients. In the 48 -h culture, $10-\mathrm{NO}_{2}$-OA significantly reduced the production of type I interferons. Interferons are known to play a crucial role in the immune host defense against viral infections [36]. In RA, the interferon signature is increased in early disease [25] and has been associated with poor response to $\mathrm{B}$ cell depleting therapy with rituximab [37]. Therefore, $10-\mathrm{NO}_{2}-\mathrm{OA}$ could be a novel option in RA patients with a high interferon signature.

Hereafter, we compared the effects of $10-\mathrm{NO}_{2}-\mathrm{OA}$ with the effects of the TNF $\alpha$ inhibitor etanercept across three ex vivo models of inflammatory arthritis. In SFMCs cultured for 48-h, 10- $\mathrm{NO}_{2}$-OA significantly reduced MCP-1 production in a dose dependent manner. The 48-h SFMC culture primarily consists of lymphocytes and monocytes. $10-\mathrm{NO}_{2}-\mathrm{OA}$ did not significantly reduce MMP3 


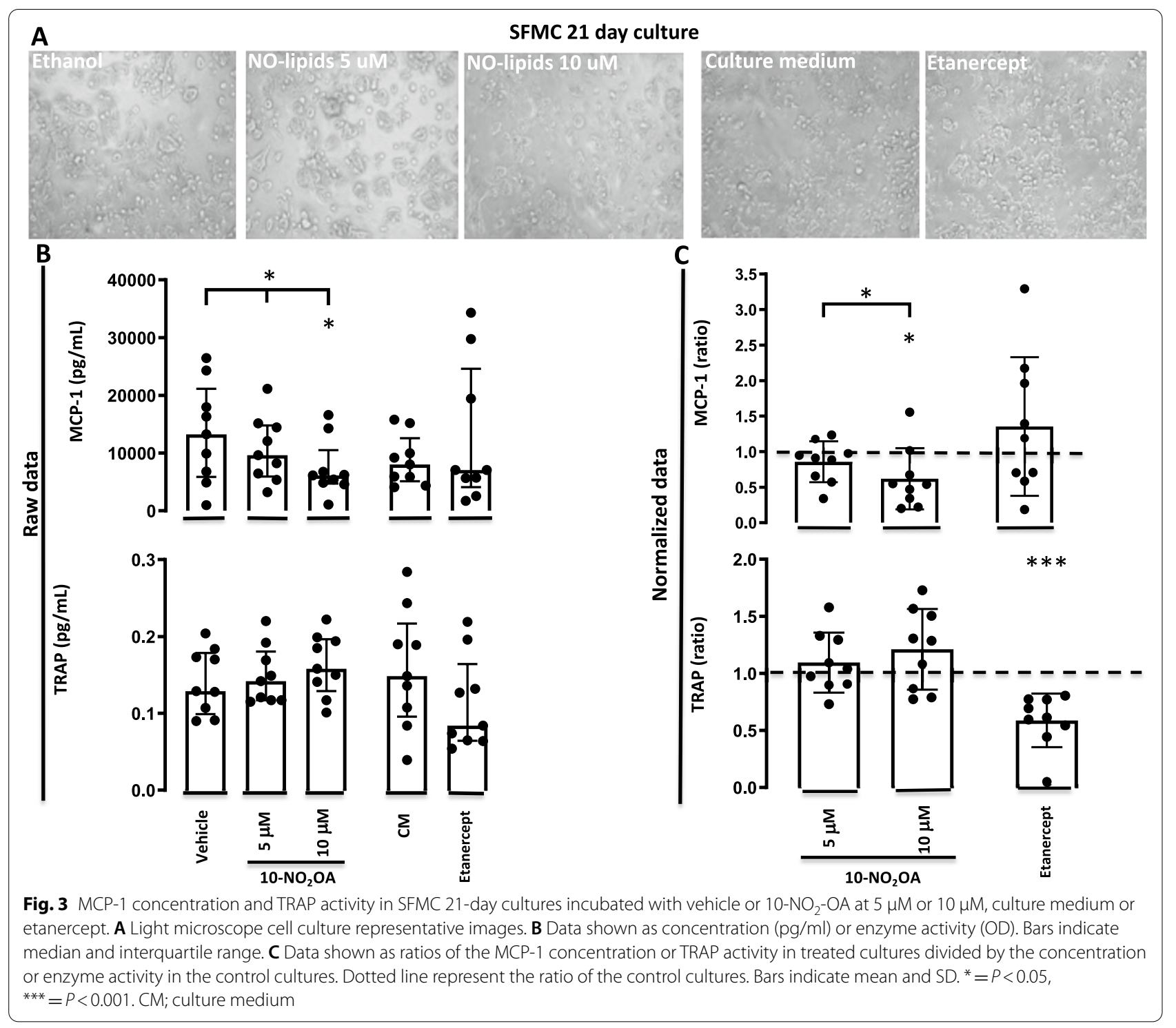

production. Etanercept decreased both the production of MCP-1 and MMP3. This indicates that $10-\mathrm{NO}_{2}-$ OA might not prevent the structural damage mediated by MMP3. However, it is not known whether other mediaters of cartilage degradation could be affected by $10-\mathrm{NO}_{2}$-OA.

The 21-day culture resembles inflammatory osteoclastogenesis. $10-\mathrm{NO}_{2}$-OA had no effect on the production of TRAP-1 in this model while etanercept decreased TRAP production. This indicates that $10-\mathrm{NO}_{2}-\mathrm{OA}$ might not prevent osteoclastogenesis in inflammatory arthritis. However, it is not known whether $10-\mathrm{NO}_{2}$-OA could influence other aspects of osteoclast activation and bone destruction. Notably, $10-\mathrm{NO}_{2}-\mathrm{OA}$ decreased MCP-1 production while etanercept had no effect on the production of MCP-1 in this model.

The FLS-PBMC co-cultures simulate the interactions of the synovial FLS with monocytes and lymphocytes. $10-\mathrm{NO}_{2}-\mathrm{OA}$ reduced the production of MCP-1 in the co-culture but not the production of MMP3. Etanercept showed no effect on either MCP-1 or MMP3 levels. This points to a possible role of using $10-\mathrm{NO}_{2}-\mathrm{OA}$ in FLSdominated inflammatory conditions.

Overall, there were several differences between $10-\mathrm{NO}_{2}-\mathrm{OA}$ and etanercept. This is interesting because it underlines that the mechanism of action is very different for the two compounds. $10-\mathrm{NO}_{2}-\mathrm{OA}$ modulates downstream signaling via NFkB, PPAR $\gamma, \mathrm{Nrf} 2 /$ Keap1, and STING. Etanercept binds and neutralizes the 


\section{FLS-PBMC 48 hour co-culture}
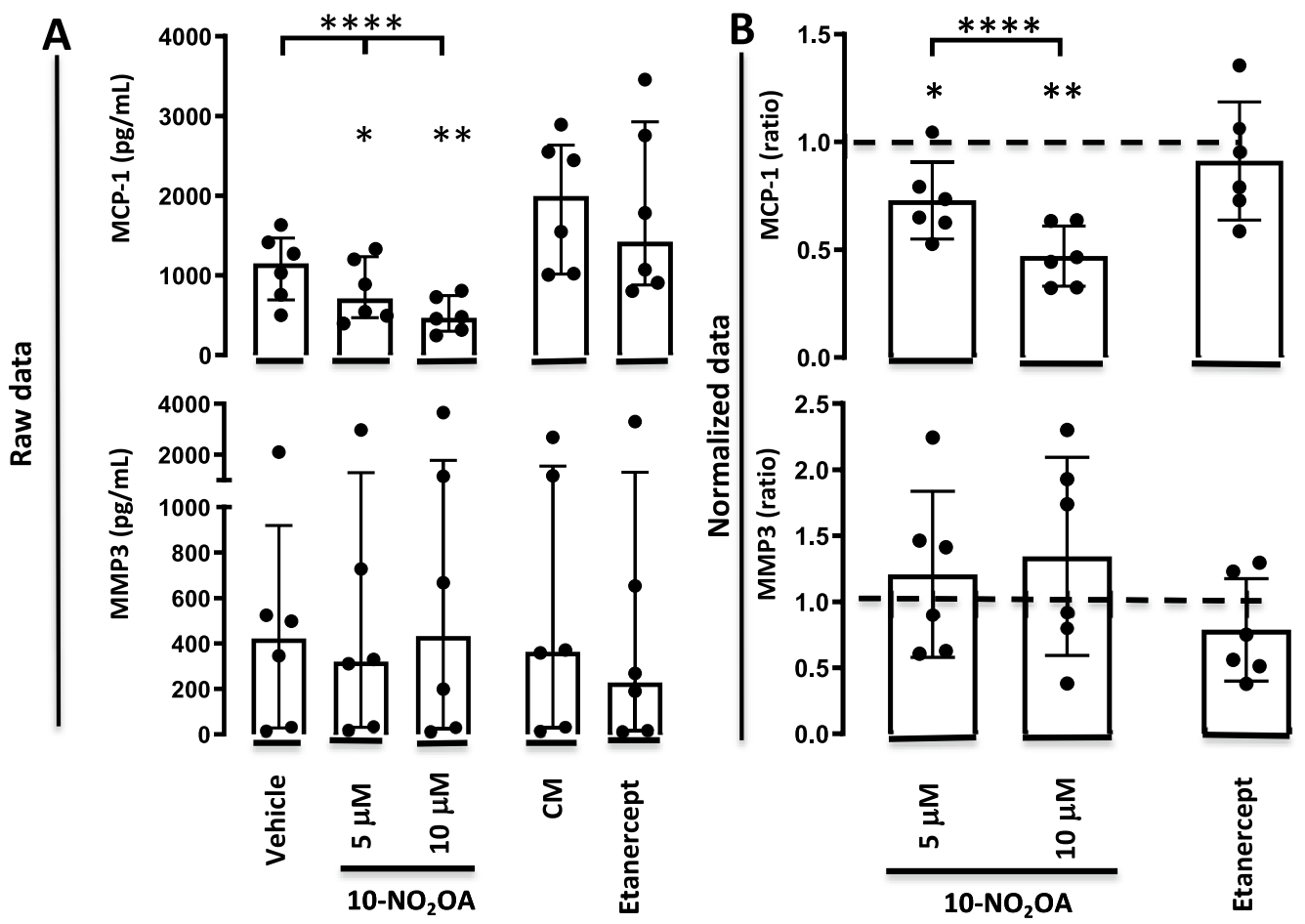

Fig. 4 MCP-1 and MMP3 concentrations in FLS-PBMC 48-h co-cultures incubated with vehicle or 10- $\mathrm{NO}_{2}-\mathrm{OA}$ at $5 \mu \mathrm{M}$ or $10 \mu \mathrm{M}$, culture medium or etanercept. A Data shown as concentrations ( $\mathrm{pg} / \mathrm{ml})$. Bars indicate median and interquartile range. B Data shown as ratios of the MCP-1 or MMP3 concentration in treated cultures divided by the concentration in the control cultures. Dotted line represent the ratio of the control cultures. Bars indicate mean and SD. ${ }^{* * *}=P<0.0001$. CM; culture medium

Table 2 Summary of results

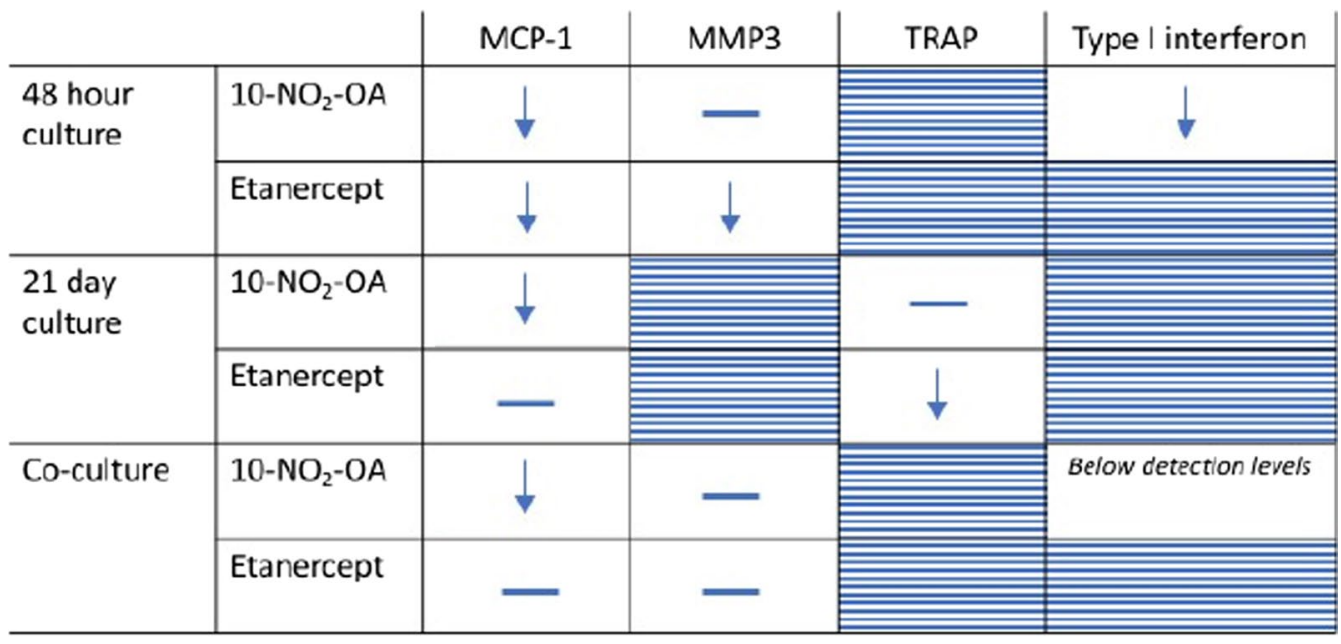

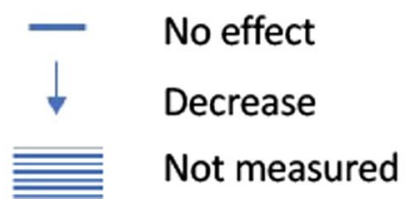

In SFMCs cultured for $48 \mathrm{~h}, 10-\mathrm{NO}_{2}-\mathrm{OA}$ decreased the secretion of bioactive type I interferons and MCP-1 but not MMP3. In SFMCs cultured for 21 days, $10-\mathrm{NO}_{2}-\mathrm{OA}$ decreased the production of MCP-1 but not TRAP. In co-cultures of FLSs and PBMCs, $10-\mathrm{NO}_{2}-\mathrm{OA}$ decreased the production of MCP-1 but not MMP3 

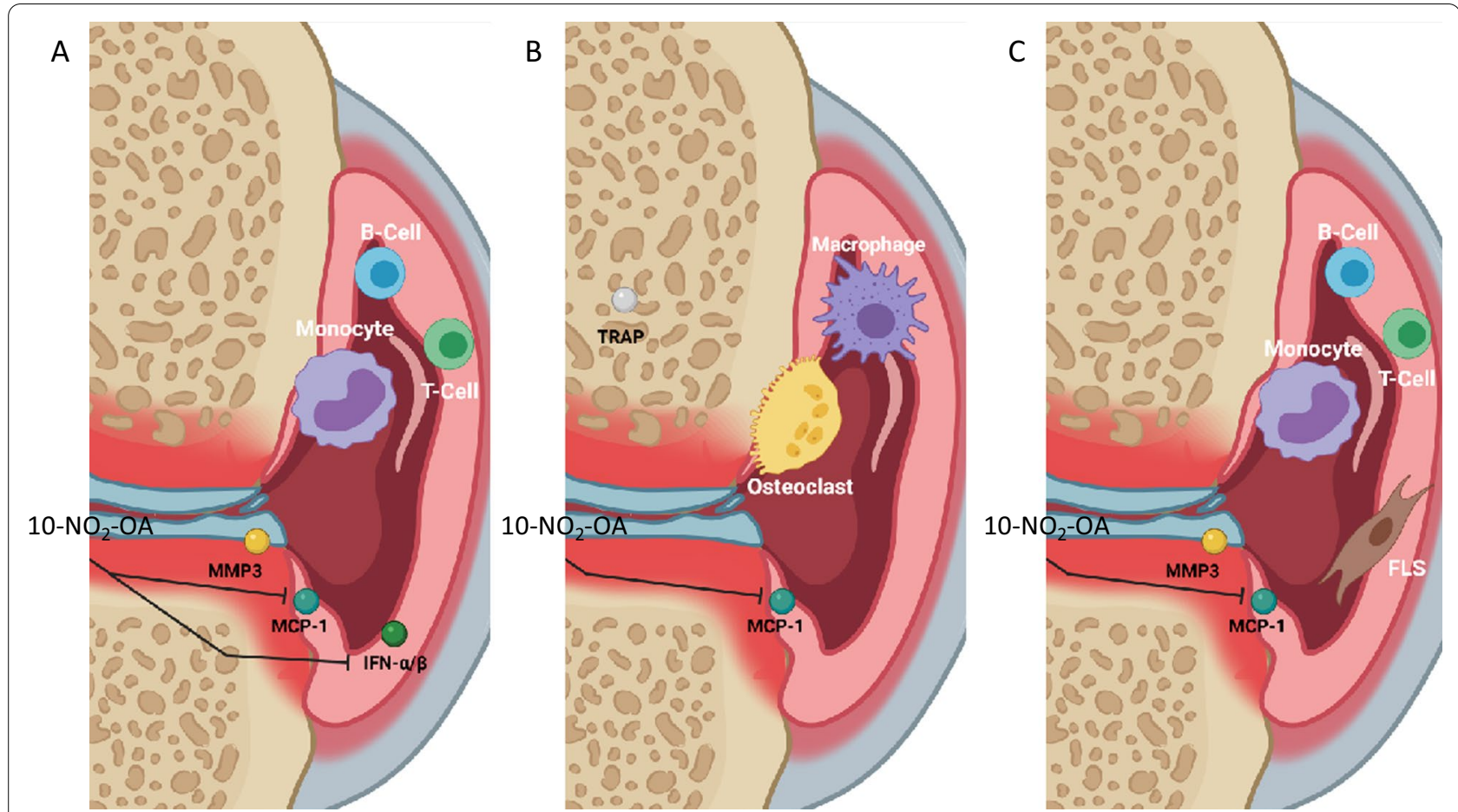

Fig. 5 Graphical summary of the results. A In SFMCs cultured for $48 \mathrm{~h}$, 10- $\mathrm{NO}_{2}-\mathrm{OA}$ decreased the secretion of bioactive type I interferons and MCP-1 but not MMP3. B In SFMCs cultured for 21 days, 10-NO $-\mathrm{OA}$ decreased the production of MCP-1 but not TRAP. C In co-cultures of FLSs and PBMCs, 10- $\mathrm{NO}_{2}-\mathrm{OA}$ decreased the production of MCP-1 but not MMP3

proinflammatory cytokine TNF $\alpha$. Therefore, $10-\mathrm{NO}_{2}$ OA could be a valueable addition in the treatment of inflammatory arthritis. Inflammatory arthritis are heterogeneous diseases. E.g., based on histological findings in synovial biopsies RA can be divided in the three pathotypes (l) lympho-myeloid, (2) diffuse-myeloid, and (3) pauci-immune characterised by prevalent stromal cells [38]. Interestingly, the pathotype dominated by FLS seemingly responds worse to treatment than other pathotypes $[39,40]$. Therefore, treatment options for this subset of patients is especially needed. Further investigations of $10-\mathrm{NO}_{2}-\mathrm{OA}$ in specifically difficult to treat RA could therefore be interesting.

There are several limitations to this study. First, we only measured type I interferons, MCP-1, MMP3, and TRAP. Many other cytokines, chemokines and enzymes are relevant in the pathogenesis of inflammatory arthritis. Further, SFMCs are rather heterogeneous with large interdonor variations. The proportion of lymphocytes and monocytes varies greatly and other cell types such as fibroblasts, endothelial cells and dendritic cells could also present. Therefore, the findings in this study are not necessarily generalizable.

To summarize, $10-\mathrm{NO}_{2}$-OA decreases $\mathrm{MCP}-1$ in three models of inflammatory arthritis (Table 2 and Fig. 5). $10-\mathrm{NO}_{2}-\mathrm{OA}$ seems to be a novel mode of action for suppressing pathogenic mechanisms in inflammatory arthritis. Interestingly, $10-\mathrm{NO}_{2}-\mathrm{OA}$ inhibited type I interferon production and $10-\mathrm{NO}_{2}-\mathrm{OA}$ was more effective in reducing MCP-1 production in cultures dominated by FLSs compared with etanercept. Therefore, our results encourage clinical investigations of $10-\mathrm{NO}_{2}-\mathrm{OA}$ in patients with inflammatory arthritis. This treatment would be a new option especially in inflammatory arthritis patients with a high interferon signature or with a fibroblast dominated pathotype.

\section{Abbreviations}

DMARD: Disease-modifying antirheumatic drug; ELISA: Enzyme-linked immunosorbent assay; FLS: Fibroblast-like synovial cell; MCP-1: Monocyte chemoattractant protein-1; MMP3: Matrix metalloproteinase 3; PBMC: Peripheral blood mononuclear cell; RA: Rheumatoid arthritis; SFMC: Synovial fluid mononuclear cell; SpA: Spondyloarthritis; TNF: Tumor necrosis factor; TRAP: Tartrate-resistant acid phosphatase.

\section{Supplementary Information}

The online version contains supplementary material available at https://doi. org/10.1186/s12865-021-00471-3.

Additional file 1. Figure S1. Flowchart showing the samples used in each experiment. Figure S2. MTT assay with synovial fluid mononuclear cells and fibroblast-like synovial cells. Figure S3. Overview of experimental setup for SFMC 48-hour and SFMC 21-day culture models. Figure S4. Overview of experimental setup for the FLS-PBMC co-culture model. 


\section{Acknowledgements}

We thank Karin Skovgaard Sørensen (Department of Biomedicine, Aarhus University) for excellent technical assistance concerning MCP-1 and MMP3 ELISA and TRAP measurement. We thank medical doctors and nurses at the Department of Rheumatology, Aarhus University Hospital for helping to collect the patient samples.

\section{Authors' contributions}

ALH, CKH and TWK helped to design the study. ALH, LSJ, ASS, MPH, SL and TWK helped to carry out the experiments and analyze and interpret the data. ALH, LSJ, ASS, and TWK helped to draft the first version of the manuscript. All authors were involved in data analysis and revising the manuscript. All authors read and approved the final manuscript.

\section{Funding}

This work was supported by Independent Research Fund Denmark (9039-00015B)

\section{Availability of data and materials}

Please contact corresponding author for data requests (email: kragstrup@ biomed.au.dk)

\section{Declarations}

\section{Ethics approval and consent to participate}

All samples were obtained after informed written consent according to the Declaration of Helsinki and approved by the Local Ethics Committee (The Central Denmark Region committee on health research ethics, Project Number 20121329) and the Danish Data Protection Agency.

\section{Consent for publication}

Not applicable.

\section{Competing interests}

TWK has engaged in educational activities receiving speaking fees from Pfizer, Bristol-Myers Squibb, Eli Lilly, Novartis, and UCB and has been consultant and advisor for Bristol-Myers Squibb and Gilead. TWK is co-owner and clinical developer in iBiotech ApS developing diagnostic and therapeutic solutions for people with autoimmune diseases and cancer. The other authors have no competing interests.

\section{Author details}

'Department of Biomedicine, Aarhus University, Høegh-Guldbergs Gade 10, C. F. Møllers Allé 6, 8000 Aarhus C, Denmark. ${ }^{2}$ Department of Rheumatology, Aarhus University Hospital, Aarhus, Denmark. ${ }^{3}$ Department of Rheumatology, Silkeborg Regional Hospital, Silkeborg, Denmark.

Received: 22 March 2021 Accepted: 2 December 2021

Published online: 17 December 2021

\section{References}

1. Taylor PC. Developing anti-TNF and biologic agents. Rheumatology (Oxford). 2011;50(8):1351-3.

2. Atzeni F, Sarzi-Puttini P, Gorla R, Marchesoni A, Caporali R. Switching rheumatoid arthritis treatments: an update. Autoimmun Rev. 2011;10(7):397-403

3. Coles B, Bloodsworth A, Clark SR, et al. Nitrolinoleate inhibits superoxide generation, degranulation, and integrin expression by human neutrophils: novel antiinflammatory properties of nitric oxide-derived reactive species in vascular cells. Circ Res. 2002;91(5):375-81.

4. O'Donnell VB, Eiserich JP, Chumley PH, et al. Nitration of unsaturated fatty acids by nitric oxide-derived reactive nitrogen species peroxynitrite, nitrous acid, nitrogen dioxide, and nitronium ion. Chem Res Toxicol. 1999;12(1):83-92.

5. Bonacci G, Baker PR, Salvatore SR, et al. Conjugated linoleic acid is a preferential substrate for fatty acid nitration. J Biol Chem. 2012;287(53):44071-82.
6. Rudolph V, Rudolph TK, Schopfer FJ, et al. Endogenous generation and protective effects of nitro-fatty acids in a murine model of focal cardiac ischaemia and reperfusion. Cardiovasc Res. 2010;85(1):155-66.

7. Vitturi DA, Minarrieta L, Salvatore SR, et al. Convergence of biological nitration and nitrosation via symmetrical nitrous anhydride. Nat Chem Biol. 2015;11(7):504-10.

8. Villacorta L, Minarrieta L, Salvatore SR, et al. In situ generation, metabolism and immunomodulatory signaling actions of nitro-conjugated linoleic acid in a murine model of inflammation. Redox Biol. 2018;15:522-31.

9. Hansen AL, Buchan GJ, Ruhl M, et al. Nitro-fatty acids are formed in response to virus infection and are potent inhibitors of STING palmitoylation and signaling. Proc Natl Acad Sci USA. 2018;115(33):E7768-75.

10. Cui T, Schopfer FJ, Zhang J, et al. Nitrated fatty acids: endogenous antiinflammatory signaling mediators. J Biol Chem. 2006;281(47):35686-98.

11. Baker PR, Lin Y, Schopfer FJ, et al. Fatty acid transduction of nitric oxide signaling: multiple nitrated unsaturated fatty acid derivatives exist in human blood and urine and serve as endogenous peroxisome proliferator-activated receptor ligands. J Biol Chem. 2005;280(51):42464-75.

12. Li Y, Zhang J, Schopfer FJ, et al. Molecular recognition of nitrated fatty acids by PPAR gamma. Nat Struct Mol Biol. 2008;15(8):865-7.

13. Kansanen E, Bonacci G, Schopfer FJ, et al. Electrophilic nitro-fatty acids activate NRF2 by a KEAP1 cysteine 151-independent mechanism. J Biol Chem. 2011;286(16):14019-27.

14. Garner RM, Mould DR, Chieffo C, Jorkasky DK. Pharmacokinetic and pharmacodynamic effects of oral CXA-10, a nitro fatty acid, after single and multiple ascending doses in healthy and obese subjects. Clin Transl Sci. 2019;12(6):667-76.

15. Ishikawa H, Barber GN. STING is an endoplasmic reticulum adaptor that facilitates innate immune signalling. Nature. 2008;455(7213):674-8.

16. Ishikawa H, Ma Z, Barber GN. STING regulates intracellular DNAmediated, type I interferon-dependent innate immunity. Nature. 2009;461(7265):788-92.

17. Burdette $D L$, Vance RE. STING and the innate immune response to nucleic acids in the cytosol. Nat Immunol. 2013;14(1):19-26.

18. Hansen K, Prabakaran T, Laustsen A, et al. Listeria monocytogenes induces IFN $\beta$ expression through an IFI16-, CGAS- and STING-dependent pathway. EMBO J. 2014;33(15):1654-66.

19. Burdette DL, Monroe KM, Sotelo-Troha K, et al. STING is a direct innate immune sensor of cyclic di-GMP. Nature. 2011;478(7370):515-8.

20. Woodward JJ, lavarone AT, Portnoy DA. c-di-AMP secreted by intracellular Listeria monocytogenes activates a host type I interferon response. Science (New York, NY). 2010;328(5986):1703-5.

21. McWhirter SM, Barbalat R, Monroe KM, et al. A host type I interferon response is induced by cytosolic sensing of the bacterial second messenger cyclic-di-GMP. J Exp Med. 2009;206(9):1899-911.

22. Mukai K, Konno H, Akiba T, et al. Activation of STING requires palmitoylation at the Golgi. Nat Commun. 2016;7:11932.

23. Hansen AL, Mukai K, Schopfer FJ, Taguchi T, Holm CK. STING palmitoylation as a therapeutic target. Cell Mol Immunol. 2019;16(3):236-41.

24. Narendra SC, Chalise JP, Hook N, Magnusson M. Dendritic cells activated by double-stranded RNA induce arthritis via autocrine type I IFN signaling. J Leukoc Biol. 2014;95(4):661-6.

25. Cooles FAH, Anderson AE, Lendrem DW, et al. The interferon gene signature is increased in patients with early treatment-naive rheumatoid arthritis and predicts a poorer response to initial therapy. J Allergy Clin Immunol. 2018;141(1):445-8.e4.

26. Bernardini G, Benigni G, Scrivo R, Valesini G, Santoni A. The multifunctional role of the chemokine system in arthritogenic processes. Curr Rheumatol Rep. 2017;19(3):11.

27. Bianconi V, Sahebkar A, Atkin SL, Pirro M. The regulation and importance of monocyte chemoattractant protein-1. Curr Opin Hematol. 2018;25(1):44-51.

28. Koch AE, Kunkel SL, Harlow LA, et al. Enhanced production of monocyte chemoattractant protein-1 in rheumatoid arthritis. J Clin Investig. 1992;90(3):772-9.

29. Chao PZ, Hsieh MS, Cheng CW, Lin YF, Chen CH. Regulation of MMP-3 expression and secretion by the chemokine eotaxin-1 in human chondrocytes. J Biomed Sci. 2011;18:86. 
30. Janckila AJ, Parthasarathy RN, Parthasarathy LK, et al. Properties and expression of human tartrate-resistant acid phosphatase isoform 5 a by monocyte-derived cells. J Leukoc Biol. 2005;77(2):209-18.

31. Kragstrup TW, Adams M, Lomholt S, et al. IL-12/IL-23p40 identified as a downstream target of apremilast in ex vivo models of arthritis. Ther Adv Musculoskelet Dis. 2019. https://doi.org/10.1177/1759720X19828669.

32. Lomholt S, Mellemkjaer A, Iversen MB, Pedersen SB, Kragstrup TW. Resveratrol displays anti-inflammatory properties in an ex vivo model of immune mediated inflammatory arthritis. BMC Rheumatol. 2018;2:27

33. Nielsen MA, Lomholt S, Mellemkjaer A, Andersen MN, Buckley CD, Kragstrup TW. Responses to cytokine inhibitors associated with cellular composition in models of immune-mediated inflammatory arthritis. ACR Open Rheumatol. 2020;2(1):3-10.

34. Greisen SR, Einarsson HB, Hvid M, Hauge EM, Deleuran B, Kragstrup TW. Spontaneous generation of functional osteoclasts from synovial fluid mononuclear cells as a model of inflammatory osteoclastogenesis. APMIS. 2015;123(9):779-86.

35. Stougaard J, Lomholt S, Ommen P, Kelsen J, Kragstrup TW. The antifibrotic drug pirfenidone inhibits spondyloarthritis fibroblast-like synoviocytes and osteoblasts in vitro. BMC Rheumatol. 2018;2:33.

36. Liu SY, Sanchez DJ, Aliyari R, Lu S, Cheng G. Systematic identification of type I and type II interferon-induced antiviral factors. Proc Natl Acad Sci USA. 2012;109(11):4239-44

37. Thurlings RM, Boumans M, Tekstra J, et al. Relationship between the type I interferon signature and the response to rituximab in rheumatoid arthritis patients. Arthritis Rheum. 2010;62(12):3607-14.

38. Townsend MJ. Molecular and cellular heterogeneity in the rheumatoid arthritis synovium: clinical correlates of synovitis. Best Pract Res Clin Rheumatol. 2014;28(4):539-49.

39. Humby F, Lewis M, Ramamoorthi N, et al. Synovial cellular and molecular signatures stratify clinical response to csDMARD therapy and predict radiographic progression in early rheumatoid arthritis patients. Ann Rheum Dis. 2019;78(6):761-72

40. Lewis MJ, Barnes MR, Blighe K, et al. Molecular portraits of early rheumatoid arthritis identify clinical and treatment response phenotypes. Cell Rep. 2019;28(9):2455-70.e5.

\section{Publisher's Note}

Springer Nature remains neutral with regard to jurisdictional claims in published maps and institutional affiliations.

Ready to submit your research? Choose BMC and benefit from:

- fast, convenient online submission

- thorough peer review by experienced researchers in your field

- rapid publication on acceptance

- support for research data, including large and complex data types

- gold Open Access which fosters wider collaboration and increased citations

- maximum visibility for your research: over $100 \mathrm{M}$ website views per year

At BMC, research is always in progress.

Learn more biomedcentral.com/submissions 